\title{
Uso de conglomerados bietapicos para la construcción de perfiles adictivos en estudiantes universitarios
}

\section{Use of bietapic conglomerates for the construction of addictive profiles in university students}

Jessica Alexandra Marcatoma Tixi. ${ }^{1}$, Héctor Salomón Mullo Guaminga. ${ }^{2}$ \& Natalia Alexandra Pérez Londo. ${ }^{3}$

Recibido: 01-08-2019 / Revisado: 05-09-209 /Aceptado: 28-09-2019/ Publicado: 04-10-2019

\begin{abstract}
. https://doi.org/10.33262/cienciadigital.v3i4.963

The academic success of the university student is negatively affected by certain addictive behaviors, which is a progressive problem and is considered an increasingly common event among university students, however, studies dealing with this problem in Ecuador are scarce. Objective. Define addiction profiles in students of the Polytechnic School of Chimborazo. Materials and methods. Cross-sectional, quantitative, non-experimental and exploratory research; It studies 945 university students from a total of 18,641, using as instruments: the MULTICAGE 4 and Assessment of the severity of dependence (SDS) surveys, with likert questions where the statistical analysis of two-stage conglomerates was applied. Results: Two well-defined profiles were found, where the first is a combination of men (244) and women (242), while the second is basically made up of women (458). Conclusions: This last profile allows to show a very interesting conclusion in the case of women, I have indicated that they in general are highly prone to suffer eating disorders, compulsive spending disorder and internet addiction, to a lesser extent they are likely to be dependent on Alcohol and
\end{abstract}

\footnotetext{
${ }^{1}$ Escuela Superior Politécnica de Chimborazo, Facultad de Ciencias, Riobamba, Ecuador, jesaly11@hotmail.com

${ }^{2}$ Escuela Superior Politécnica de Chimborazo, Facultad de Ciencias, Riobamba, Ecuador, shalo1028@hotmail.com

${ }^{3}$ Escuela Superior Politécnica de Chimborazo, Facultad de Ciencias, Riobamba, Ecuador, nperez@espoch.edu.ec
} 
marijuana use, in contrast, are discreetly impaired by pathological gambling, substance addiction, video games and sex addiction.

Keywords: Education, Addictions, Clusters, Multicage and SDS.

\section{Resumen.}

El éxito académico del estudiante universitario se ve afectado negativamente por ciertas conductas adictivas, que es un problema progresivo y se considera un evento cada vez más habitual entre los universitarios, sin embargo, son escasos los estudios que tratan sobre esta problemática en Ecuador. Objetivo. Definir perfiles de adicción en estudiantes de la Escuela Superior Politécnica de Chimborazo. Materiales y métodos. La investigación de tipo transversal, cuantitativa, no experimental y exploratorio; estudia a 945 universitarios de un total de 18 641, empleando como instrumentos: las encuestas MULTICAGE 4 y Valoración de la gravedad de la dependencia (SDS), con preguntas de tipo likert donde se aplicó el análisis estadístico de conglomerados en dos etapas. Resultados: Se encontró dos perfiles bien definidos, donde el primero es una combinación de hombres (244) y mujeres (242), mientras que el segundo esta básicamente constituido por mujeres (458). Conclusiones: Este último perfil permite mostrar una conclusión muy interesante para el caso de las mujeres, he indica que ellas en general son altamente propensas a sufrir trastornos alimenticios, desorden de gasto compulsivo y adicción al internet, en menor medida son propensas a ser dependientes del alcohol y al consumo de marihuana, en contraste, son discretamente perjudicadas por el juego patológico, adicción a sustancias, videojuegos y adicción al sexo.

Palabras claves: Educación, Adicciones, Conglomerados en dos fases, Multicage y SDS.

\section{Introducción.}

El éxito académico del estudiante universitario está ligado al cumplimiento satisfactorio de la carga horaria, responsabilidades académicas, tareas, organización del estudio, evaluaciones, entre otros (Dominguez, Villegas y Centeno, 2014) (Ramos, Jadán, Paredes, Bolaños y Gómez, 2017). Aquellos que cumplen con dicho perfil son denominados estudiantes de alto rendimiento o exitosos, sin embargo, ésta no es una realidad absoluta, ya que existe un elevado porcentaje de estudiantes que no son capaces de tener éxito académico, entre otras cosas debido a que, desarrollan adicciones que reducen el rendimiento académico (Ramos et al., 2017). Estas adicciones están presentes en el ámbito universitario en general, por ejemplo en la Universidad de la Amazonia de Colombia, el 95\% de estudiantes consumen alcohol (Salgado, Aldana y Gaitán, 2018), en la Facultad de Ciencias Empresarias de la Universidad San Ignacio de Loyola, existe una alta frecuencia de uso de redes sociales por parte de los alumnos(Palacios, 2018), igualmente, en la Facultad de Educación de Albacete, más de la mitad de los estudiantes dedican más de cinco 
horas al día al teléfono móvil y de estos el 79,6\% manifiestan haber tenido problemas secundarios de comportamiento por su uso excesivo (Garrote, Jiménez y Gómez, 2018), en el Centro Universitario de Ciencias Biológicas y Agrícolas de la Universidad de Guadalajara, hay 26,7\% de fumadores, además el 12,8\% de las personas encuestadas consumió drogas ilegales en el último mes y el 23,8\% en el último año, donde la principal droga ilegal utilizada fue la marihuana (Gómez, Landeros, Pérez y Martínez, 2018). Estos comportamientos, se dan tal vez debido a que los estudiantes piensan en la adicción desde la posibilidad de la elección, al tiempo que lo asocian con el riesgo del daño; así mismo, a causa de que cuestionan el perjuicio social e institucional de estas conductas en el contexto universitario (Osorio, Díez y Bedoya, 2018).

Por tanto, el tema de las adicciones son motivo de investigaciones sociales, económicas y científicas ya que es un síndrome constituido por un conjunto de signos y síntomas en la sociedad a nivel mundial, actualmente la mayoría de los estudios centran su atención sobre colectivos universitarios debido al deterioro progresivo de la calidad de vida de los estudiantes a través de la pérdida de control, negación o auto engaño y soledad. Estos son algunos de los síntomas característicos de las adicciones cuya presencia impide percibir el deterioro personal a causa de una conducta adictiva, estos patrones se relacionan con cambios neuroquímicos presentes en personas con desordenes adictivos con cierta predisposición biogenética a desarrollar estas enfermedades.

En la actualidad varias son las adicciones que afectan a la población universitaria, como el consumo de alcohol, adicción a los juegos de azar, consumo de sustancias, trastornos alimenticios, adicción al uso de internet, gasto compulsivo, sexo, uso de celular, consumo de cigarrillo y marihuana (López, 2019), las cuales empiezan a manifestarse de forma creciente, esto se relaciona con la Teoría de Normas Sociales donde explican acerca de la influencia que ejercen particularmente amigos y compañeros en la adquisición de adicciones, el punto de partida de estas enfermedades es la búsqueda de aceptación social donde existen grupos referentes de alto dominio social en el que las normas son implícitas, impuestas por unos pocos y no abiertamente discutidas, lo cual lleva a que los jóvenes adquieran formas de pensamiento, sin necesariamente concordar con ellas.

Dentro de las adicciones en el país (INEC, 2012) (Brito, 2018), el grupo con mayor consumo de alcohol se encuentra entre las edades de 19 a 24 años que son el 12\% de la población ecuatoriana, por otro lado, la Organización Mundial de la Salud (OMS) en el año 2013, manifiesta que Ecuador ocupa el segundo lugar en América Latina en consumo de alcohol, esto evidencia que en el país el consumo de bebidas alcohólicas predomina entre las adicciones, reflejando una sociedad sumida en una cultura de alcohol, esta problemática afecta más a los hogares con menores ingresos, que según estimaciones del INEC gastan en conjunto más de 545 mil dólares al mes, obviamente aquellos con mayores ingresos les superan, empleando más de 2 millones 130 mil dólares para su consumo, pues cada habitante ingiere en promedio 9.4 litros de alcohol al año (INEC, 2015) (Túquerres y Ulcuango, 2019). De la misma manera, estudios recientes revelan que en dos universidades de la capital del Ecuador, existe un uso y abuso del internet, ya que en este, se 
encuentran plataformas virtuales de aprendizaje, donde el estudiante debe subir tareas e interactuar para aprender, pero también se encuentran otras páginas, donde el estudiante podría desperdiciar su tiempo con el uso de redes sociales, juegos en línea, reproductores de películas, entre otros recursos de la web, que se convierten en estímulos que aumentan el comportamiento adictivo del estudiante universitario. Por otro lado, el uso de internet ha incentivado la adicción al sexo virtual, esto fue confirmado por estadísticas proporcionadas por Google a través de los motores de búsqueda, donde la palabra más buscada fue sexo (Ramos et al., 2017) (Parra, Vargas, Zamorano, Peña, Velázquez, Ruiz y Monreal, 2016) (García, Terol, Nieto, Lledó, Sánchez, Martín y Sitges, 2008) (Ndege, Mutavi, Kokonya, Nekesa, Musungu, Obondo y Wangari, 2015). Otra adicción es el consumo de marihuana, preocupación central desde el punto de vista de las políticas públicas, pues se identifica como puerta de entrada hacia otras sustancias que originan trayectorias de consumo problemáticas y consecuencias negativas para los proyectos de vida de personas jóvenes, especialmente cuando su consumo aparece como algo cotidiano dentro del espacio universitario (Cazenave, Saavedra, Huerta, Mendoza y Aguirre, 2017). Además, el consumo de imágenes impuestas por el medio social y familiar coadyuva a que un estudiante padezca de algún tipo de trastorno alimenticio, marcando una nueva adición por la preocupación del estándar de cuerpo perfecto generando un uso abusivo de laxantes y en ocasiones de diuréticos. En atención a lo cual, y sabiendo que en el Ecuador existen, 3173614 jóvenes entre 18 y 29 años que corresponde al $18.4 \%$ de la población ecuatoriana (Palacios, 2018) y que estos son los más vulnerables de sufrir adicciones, la presente investigación busca definir perfiles de adicción en estudiantes de la Escuela Superior Politécnica de Chimborazo (ESPOCH), que alberga aproximadamente a 18641 jóvenes universitarios de casi todas las provincias del país.

El resto del documento está organizado de la siguiente manera: en la Sección 2: revisión breve de los materiales y métodos utilizados para alcanzar el objetivo planteado, Sección 3: descripción de los principales resultados sobre los perfiles de adicción de estudiantes universitarios. Al termino la sección 4 muestra las conclusiones de la investigación y sugerencias para futuras investigaciones.

\section{Metodología}

Esta investigación fue transversal, cuantitativa, no experimental y exploratoria, según la línea del tiempo, tipo de investigación, manipulación de variables y profundidad del objetivo de estudio respectivamente (Hernández, Fernández y Baptista, 2014) (Patten y Newhart, 2017).

Instrumentos de recolección de datos: La encuesta aplicada mantuvo como temática principal las adicciones, contó con un total de 34 preguntas divididas en diferentes bloques. Todas las respuestas que se recogieron fueron de tipo categórico en su mayoría con escala tipo Likert. La pregunta inicial hace referencia al sexo de los universitarios, seguido por un bloque de 32 preguntas referentes a la Dependencia del Alcohol, Juego Patológico, Adicción a Sustancias, Trastornos 
Alimenticios, Adicción a Internet, Adicción a Videojuegos, Gasto Compulsivo y Adicción al Sexo, planteadas según el cuestionario MULTICAGE 4 (Pedrero, Rodríguez, Gallardo, Fernández, Pérez y Chicharro, 2007) (Marcatoma, 2016), cuya fiabilidad es de 0,7. Mientras que el bloque final recogió datos del Consumo de Marihuana, siguiendo el cuestionario de Valoración de la gravedad de la dependencia SDS por sus siglas en inglés (Marcatoma, 2016) (Van Der Pol, Liebregts, R. de Graaf, Korf, Brink y Laar, 2013).

Población: El marco referencial de estudio estuvo representado por la totalidad (18 641) de estudiantes de la Escuela Superior Politécnica de Chimborazo (Palacios, 2018), matriculados en el período académico septiembre 2018 - febrero 2019.

Diseño Muestral: La técnica de selección de datos fue el muestreo de conglomerados bietápico donde el marco referencial de la primera etapa estuvo representado por las 38 carreras ofrecidas por la ESPOCH, de estas, se seleccionaron las carreras de Ingeniería en Administración de Empresas, Licenciatura en Promoción de la Salud, Licenciatura en Diseño Gráfico y BioquímicaFarmacia (llegado a una muestra de 945 universitarios); luego en la segunda etapa se realizó un censo en cada conglomerado, tomado como unidades de observación los niveles (o cursos) de las diferentes carreras. Es importante mencionar que el tamaño de la muestra se determinó fijando el error máximo admisible para estimar una proporción en muestreo por conglomerados con afijación proporcional a un $95 \%$ de confianza, garantizando la representatividad de la muestra y las estimaciones en base a ella.

Localización del estudio: El estudio se desarrolló en el campus Riobamba de la Escuela Superior Politécnica de Chimborazo.

Técnicas estadísticas: Dada la utilidad del empleo de técnicas estadísticas para el análisis cuantitativo de fenómenos sociales, en esta investigación se ha seleccionado el análisis de conglomerados en dos etapas con el propósito de profundizar en la compresión de las diferentes agrupaciones de los estudiantes de la ESPOCH en función de variables de adicción. Pérez, 2005 (como se citó en Mullo y Marcatoma, 2018) indica que el procedimiento, análisis de conglomerados en dos fases de SPSS es una herramienta de exploración diseñada para descubrir las agrupaciones naturales de un conjunto de datos que, de otra manera, no sería posible detectar. Este procedimiento trabaja tanto con variables continuas como categóricas. Los casos representan los objetos que se van a conglomerar y las variables representan los atributos en los que se va a basar la conglomeración. La medida de distancia de verosimilitud supone que las variables del modelo de conglomerados son independientes. Además, se supone que cada variable continua tiene una distribución normal (de Gauss) y que cada variable categórica tiene una distribución multinomial. Sin embargo, las comprobaciones empíricas internas indican que este procedimiento es bastante robusto frente a las violaciones tanto del supuesto de independencia como de las distribuciones, pero aun así es preciso tener en cuenta hasta qué punto se cumplen estos supuestos. En cuanto a la justificación de su uso, es debido principalmente a que las variables de adicción son 
de tipo categóricas y también a causa de que se pretende estudiar las agrupaciones naturales de los estudiantes universitarios con relación a variables de adicción.

\section{Resultados}

Las conductas adictivas son un problema progresivo y se consideran un evento cada vez más habitual entre los universitarios. Hace poco la bibliográfica científica está hallando un patrón de concurrencia de uso de drogas, como por ejemplo el uso desmedido del alcohol, cannabis y tabaco, pronostica el consumo de otras drogas, acompañadas por otras conductas adictivas (Mínguez y Becoña, 2015). Lo cual es importante, ya que estás conductas están originando múltiples casos de dependencia en gente que obtiene de estas adicciones un escondite, que los aparta de sus adversidades personales o familiares, pero luego no pueden salir fácilmente de estas conductas. Como se ve las adicciones son un tema interesante donde vale la pena mostrar datos actualizados sobre esta problemática, de modo que, presentamos los resultados de este estudio en dos bloques, el primero trata sobre la indagación del cumplimiento de los supuestos para la aplicación del análisis de conglomerados en dos fases y luego mostramos los resultados.

Sobre el cumplimiento de supuestos: Antes de presentar los resultados es trascendente indicar que para la comprobación de independencia y distribución multinomial de las variables en estudio, se utilizaron tablas de contingencia y pruebas chi-cuadrada respectivamente, obteniendo para las 45 combinaciones de pares de variables (Sexo, Dependencia del Alcohol, Juego Patológico, Adicción a Sustancias, Trastornos Alimenticios, Adicción a Internet, Adicción a Videojuegos, Gasto Compulsivo, Adicción al Sexo y Consumo de Marihuana), valores de p inferiores a 0.05, lo cual muestra que existe independencia entre las variables. Luego al estudiar la distribución de las variables se obtuvo valores de p superiores a 0.05 , indicando obviamente que se cumple con el supuesto de distribución multinomial.

\section{Perfiles adictivos en estudiantes universitarios de la ESPOCH}

Tabla 1. Distribución de perfiles

\begin{tabular}{ccc}
\hline Perfil & ni & fi \\
\hline 1 & 486 & $51.45 \%$ \\
2 & 459 & $48.60 \%$ \\
\hline Total & 945 & $100 \%$ \\
\hline
\end{tabular}

Fuente: Elaborado por los autores según la Encuesta de Perfiles Adictivos en la ESPOCH 


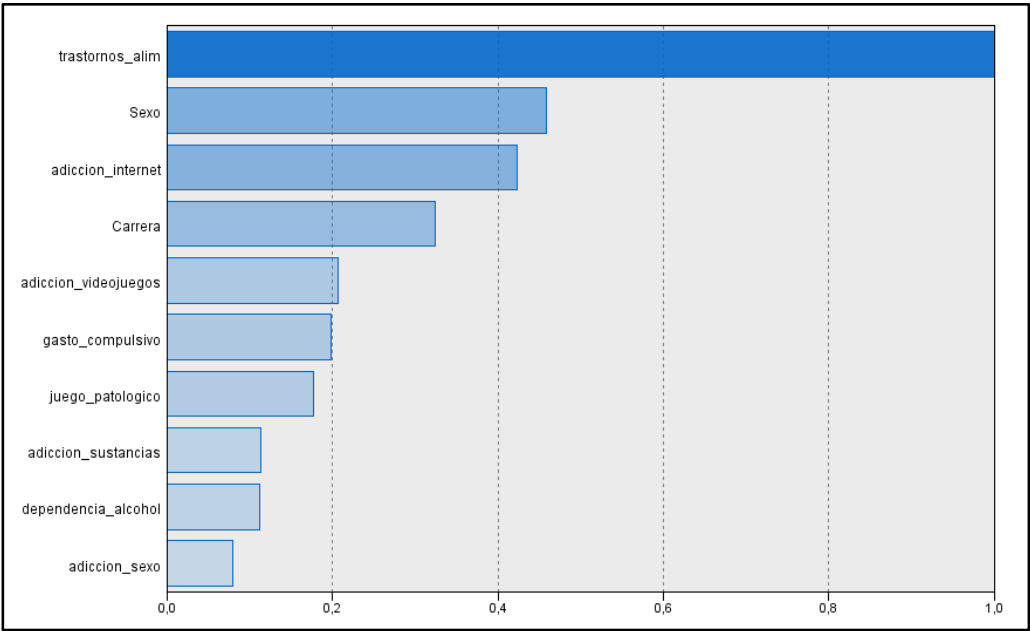

Figura 1. Importancia del Predictor

Fuente: Elaborado por los autores según la Encuesta de Perfiles Adictivos en la ESPOCH

La distribución de los perfiles (ver tabla 1.) encontrados, muestra que los estudiantes conforman 2 perfiles, el primero con 486 universitarios (51.45\%) y el segundo con 459 (48.6\%). Dentro de la importancia de las variables predictores (ver Figura. 2.) "trastornos de alimentación" es la más representativa para la clasificación y creación de los perfiles, seguida de "sexo" y "adicción a internet", también existe una homogeneidad entre las variables "adicción a sustancias" y "dependencia al alcohol". En contraste, las demás variables no aportan significativamente a la creación de los conglomerados.

Tabla 2. Distribución de perfiles (DP) según el sexo

\begin{tabular}{ccccc}
\hline \multirow{2}{*}{ Perfil } & \multicolumn{2}{c}{ Hombre } & \multicolumn{2}{c}{ Mujer } \\
\cline { 2 - 5 } & ni & fi & ni & fi \\
\hline 1 & 244 & $99.6 \%$ & 242 & $34.6 \%$ \\
2 & 1 & $0.4 \%$ & 458 & $65.4 \%$ \\
\hline Total & 245 & $100 \%$ & 700 & $100 \%$ \\
\hline
\end{tabular}

Fuente: Elaborado por los autores según la Encuesta de Perfiles Adictivos en la ESPOCH

Tabla 3. DP según dependencia al alcohol

\begin{tabular}{ccccccccc}
\hline \multirow{2}{*}{ Perfil } & \multicolumn{2}{c}{ I } & \multicolumn{2}{c}{ PE } & \multicolumn{2}{c}{ MPE } & \multicolumn{2}{c}{ SE } \\
\cline { 2 - 9 } & ni & fi & ni & fi & ni & fi & ni & fi \\
\hline 1 & 188 & $49.90 \%$ & 154 & $41.20 \%$ & 78 & $61.40 \%$ & 66 & $98.50 \%$ \\
2 & 189 & $50.10 \%$ & 220 & $58.80 \%$ & 49 & $38.60 \%$ & 1 & $1.50 \%$ \\
\hline Total & 377 & $100 \%$ & 374 & $100 \%$ & 127 & $100 \%$ & 67 & $100 \%$ \\
\hline
\end{tabular}

Fuente: Elaborado por los autores según la Encuesta de Perfiles Adictivos en la ESPOCH 
La distribución de los perfiles en las variables de adicción examina el grado de dependencia según inexistencia (I), posible existencia (PE), muy probable existencia (MPE) y segura existencia (SE). En relación con la variable sexo, se encontró que el primer perfil está constituido (ver Tabla. 2.) por hombres $(244,99.6 \%)$ y mujeres $(242,34.6 \%)$ casi en la misma cantidad, no obstante, el segundo perfil está compuesto básicamente por mujeres $(458,65.4 \%)$. Es interesante observar que según la dependencia al alcohol (ver Tabla. 3.) de los estudiantes, el primer perfil agrupa a aquellos que son propensos (muy probable existencia y segura existencia) a ser dependientes del alcohol $(78,61.4 \%$ y $66,98.5 \%)$, en contraste en el segundo perfil, están quienes tienen nula o posible existencia $(220,58.8 \%$ y $189,50.1 \%)$ de ser dependientes del alcohol. Esto se debe a que generalmente los hombres son aquellos que consumen en mayor medida alcohol, pero recientemente las mujeres han incursionado en esta problemática y es por ello que en el primer perfil hay una mezcla entre hombres y mujeres. Por otro lado, las mujeres más conservadoras están en el segundo perfil.

Tabla 4. DP según juego patológico

\begin{tabular}{ccccccc}
\hline \multirow{2}{*}{ Perfil } & \multicolumn{2}{c}{ I } & \multicolumn{2}{c}{ PE } & \multicolumn{2}{c}{ MPE } \\
\cline { 2 - 7 } & ni & fi & ni & fi & ni & fi \\
\hline 1 & 368 & $44.60 \%$ & 105 & $98.10 \%$ & 13 & $100 \%$ \\
2 & 457 & $55.40 \%$ & 2 & $1.90 \%$ & 0 & $0 \%$ \\
\hline Total & 825 & $100 \%$ & 107 & $100 \%$ & 13 & $100 \%$
\end{tabular}

Fuente: Elaborado por los autores según la Encuesta de Perfiles Adictivos en la ESPOCH

Según juego patológico ${ }^{4}$, el primer componente engloba a las personas que tienen posible y muy probable existencia $(105,98.1 \%$ y $13,100 \%)$ de sufrir trastornos del control de impulsos hacia los juegos (ver Tabla. 4.). Esto indica que análogo a lo que ocurrió en dependencia al alcohol los hombres son aquellos que tienen tendencias a sufrir de estos males, de igual modo (ver Tabla. 5.) en lo referente al tema de adicción a sustancias $(39,100 \%$ y 2, 100\%). En cambio, el perfil dos (ver Tablas. 4. y 5.) que recordamos está conformado por mujeres, tiende a mostrar una propensión a la inexistencia $(457,55.4 \%)$ de trastornos del control de los impulsos hacia los juegos y al mismo tiempo inexistencia y posible existencia $(382,47.2 \%$ y $77,81.9 \%)$ de adicción a sustancias.

Tabla 5. DP según adicción a sustancias

\begin{tabular}{ccccccccc}
\hline \multirow{2}{*}{ Perfil } & \multicolumn{2}{c}{ I } & \multicolumn{2}{c}{ PE } & \multicolumn{2}{c}{ MPE } & \multicolumn{2}{c}{ SE } \\
\cline { 2 - 9 } & ni & fi & ni & fi & ni & fi & ni & fi \\
\hline 1 & 428 & $52.80 \%$ & 17 & $18.10 \%$ & 39 & $100 \%$ & 2 & $100 \%$ \\
2 & 382 & $47.20 \%$ & 77 & $81.90 \%$ & 0 & $0 \%$ & 0 & $0 \%$ \\
\hline Total & 810 & $100 \%$ & 94 & $100 \%$ & 39 & $100 \%$ & 2 & $100 \%$ \\
\hline
\end{tabular}

Fuente: Elaborado por los autores según la Encuesta de Perfiles Adictivos en la ESPOCH

\footnotetext{
${ }^{4}$ El juego patológico es un trastorno del control de los impulsos, que se caracteriza por una frecuente y creciente preocupación por el juego de forma desadaptativa.
} 
Tabla. 6. DP según trastorno de alimentación

\begin{tabular}{ccccccccc}
\hline \multirow{2}{*}{ Perfil } & \multicolumn{2}{c}{ I } & \multicolumn{2}{c}{ PE } & \multicolumn{2}{c}{ MPE } & \multicolumn{2}{c}{ SE } \\
\cline { 2 - 9 } & ni & fi & ni & fi & ni & fi & ni & fi \\
\hline 1 & 403 & $100 \%$ & 58 & $29.40 \%$ & 23 & $7.00 \%$ & 2 & $11.10 \%$ \\
2 & 0 & $0 \%$ & 139 & $70.60 \%$ & 304 & $93.00 \%$ & 16 & $88.90 \%$ \\
\hline Total & 403 & $100 \%$ & 197 & $100 \%$ & 327 & $100 \%$ & 18 & $100 \%$ \\
\hline
\end{tabular}

Fuente: Elaborado por los autores según la Encuesta de Perfiles Adictivos en la ESPOCH

El trastorno de alimentación (ver Tabla. 6.), generalmente es más característico en mujeres, pero se conocen casos de ambos sexos, esto ocurre en los estudiantes de la ESPOCH, donde las mujeres que se enmarcan en el segundo perfil son aquellas que tiene la mayor probabilidad de padecer de trastorno (304, 93\% y 16, 88.9\%), sin embargo, en el perfil uno donde hay una combinación de hombres y mujeres la incidencia de trastorno de alimentación es inexistente o posiblemente existente $(403,100 \%$ y 58, 29.4\%), lo cual demuestra lo dicho al principio de este párrafo.

Tabla 7. DP según adicción a internet

\begin{tabular}{ccccccccc}
\hline \multirow{2}{*}{ Perfil } & \multicolumn{2}{c}{ I } & \multicolumn{2}{c}{ PE } & \multicolumn{2}{c}{ MPE } & \multicolumn{2}{c}{ SE } \\
\cline { 2 - 9 } & ni & fi & ni & fi & ni & fi & ni & fi \\
\hline 1 & 188 & $100 \%$ & 91 & $29.30 \%$ & 162 & $40.60 \%$ & 45 & $95.70 \%$ \\
2 & 0 & $0 \%$ & 220 & $70.70 \%$ & 237 & $59.40 \%$ & 2 & $4.30 \%$ \\
\hline Total & 188 & $100 \%$ & 311 & $100 \%$ & 399 & $100 \%$ & 47 & $100 \%$ \\
\hline
\end{tabular}

Fuente: Elaborado por los autores según la Encuesta de Perfiles Adictivos en la ESPOCH

De acuerdo con la adicción a internet (ver Tabla. 7.) el primer perfil tiene una combinación de personas que por un lado tienen una nula posibilidad $(188,100 \%)$ de tener adicción a internet y por otro lado aquellos que tienen segura existencia $(45,95.7 \%)$ de experimentar adicción, en cambio en el segundo perfil están aquellos que tienen un riesgo medio hacia esta problemática, es decir, presentan posible existencia o muy probable existencia de adicción a internet (220, 70.7\% y $237,59.4 \%$ ). Esto tal vez se deba a que el segundo perfil está conformado por mujeres y el primero por una combinación de hombres y mujeres.

Sabiendo que la producción de videojuegos está exclusivamente enfocada hacia hombres, con publicidad que hace que se sientan placenteros, e incluso usándolos muy frecuentemente como el sujeto video jugador por excelencia. Hace que en el primer perfil (ver Tabla. 8.) haya muy probable y segura existencia $(40,100 \%$ y 19, 100\%) de dependencia hacia los videojuegos y en el segundo no $(456,56.9 \%$ y $3,3.6 \%)$. 
Tabla 8. DP según adicción a videojuegos

\begin{tabular}{ccccccccc}
\hline \multirow{2}{*}{ Perfil } & \multicolumn{2}{c}{ I } & \multicolumn{2}{c}{ PE } & \multicolumn{2}{c}{ MPE } & \multicolumn{2}{c}{ SE } \\
\cline { 2 - 8 } & ni & fi & ni & fi & ni & fi & ni & fi \\
\hline 1 & 346 & $43.10 \%$ & 81 & $96.40 \%$ & 40 & $100 \%$ & 19 & $100 \%$ \\
2 & 456 & $56.90 \%$ & 3 & $3.60 \%$ & 0 & $0 \%$ & 0 & $0 \%$ \\
\hline Total & 802 & $100 \%$ & 84 & $100 \%$ & 40 & $100 \%$ & 19 & $100 \%$ \\
\hline
\end{tabular}

Fuente: Elaborado por los autores según la Encuesta de Perfiles Adictivos en la ESPOCH

Tabla 9. DP según gasto compulsivo

\begin{tabular}{ccccccccc}
\hline \multirow{2}{*}{ Perfil } & \multicolumn{2}{c}{ I } & \multicolumn{2}{c}{ PE } & \multicolumn{2}{c}{ MPE } & \multicolumn{2}{c}{ SE } \\
\cline { 2 - 9 } & ni & fi & ni & fi & ni & fi & ni & fi \\
\hline 1 & 313 & $62.00 \%$ & 75 & $29.60 \%$ & 44 & $33.10 \%$ & 54 & $100 \%$ \\
2 & 192 & $38.00 \%$ & 178 & $70.40 \%$ & 89 & $66.90 \%$ & 0 & $0 \%$ \\
\hline Total & 505 & $100 \%$ & 253 & $100 \%$ & 133 & $100 \%$ & 54 & $100 \%$ \\
\hline
\end{tabular}

Fuente: Elaborado por los autores según la Encuesta de Perfiles Adictivos en la ESPOCH

Según gasto compulsivo (ver Tabla. 9.) el primer componente está conformado por estudiantes que no tienen tendencia a gastar apremiantemente $(313,62 \%)$ y también por quienes sí la tienen $(54,100 \%)$. Este último se explica debido a que comprar compulsivamente afecta primordialmente al sexo femenino y recordamos que el primer perfil es una mezcla de mujeres y hombres. En el segundo componente están fundamentalmente mujeres que tienen, si bien posible $(178,70.4 \%)$ o muy posible $(89,66.9 \%)$ tendencia a gastar compulsivamente.

La adicción al sexo es un tema sensible, entre otras cosas debido a cuestiones culturales y de miedo a ser perjudicados/as por ello, en relación con esto y teniendo presente que el segundo perfil es de mujeres y el primero de hombres y mujeres. Tenemos para el primer perfil (ver Tabla. 10.), que es posible o muy probable la existencia de sufrir adicción al sexo $(37,100 \%$ y $17,100 \%)$ y para el segundo perfil la posibilidad es nula $(459,51.5 \%)$, esto tal vez debido a que es habitual que las mujeres no se declaren adictas.

Sobre el consumo de marihuana (ver Tabla. 11.) es bien sabido que es más prevalente en hombres, esto mismo se puede observar en el perfil uno, donde existe por un lado segura existencia (13, $100 \%$ ) de consumo de marihuana y por otro lado posible existencia $(217,55.1 \%)$ de este riesgo. En contraste, en el segundo perfil el consumo de marihuana pasa de muy probable $(12,80 \%)$ a inexistente $(270,51.6 \%)$. 
Tabla 10. DP según adicción al sexo

\begin{tabular}{ccccccc}
\hline \multirow{2}{*}{ Perfil } & \multicolumn{2}{c}{ I } & \multicolumn{2}{c}{ PE } & \multicolumn{2}{c}{ MPE } \\
\cline { 2 - 7 } & ni & fi & ni & fi & ni & fi \\
\hline 1 & 432 & $48.50 \%$ & 37 & $100 \%$ & 17 & $100 \%$ \\
2 & 459 & $51.50 \%$ & 0 & $0 \%$ & 0 & $0 \%$ \\
\hline Total & 891 & $100 \%$ & 37 & $100 \%$ & 17 & $100 \%$ \\
\hline
\end{tabular}

Fuente: Elaborado por los autores según la Encuesta de Perfiles Adictivos en la ESPOCH

Tabla 11. DP según consumo de marihuana

\begin{tabular}{ccccccccc}
\hline \multirow{2}{*}{ Perfil } & \multicolumn{2}{c}{ I } & \multicolumn{2}{c}{ PE } & \multicolumn{2}{c}{ MPE } & \multicolumn{2}{c}{ SE } \\
\cline { 2 - 9 } & ni & fi & ni & fi & ni & fi & ni & fi \\
\hline 1 & 253 & $48.40 \%$ & 217 & $55.10 \%$ & 3 & $20.00 \%$ & 13 & $100 \%$ \\
2 & 270 & $51.60 \%$ & 177 & $44.90 \%$ & 12 & $80.00 \%$ & 0 & $0 \%$ \\
\hline Total & 523 & $100 \%$ & 394 & $100 \%$ & 15 & $100 \%$ & 13 & $100 \%$
\end{tabular}

Fuente: Elaborado por los autores según la Encuesta de Perfiles Adictivos en la ESPOCH

\section{Discusión}

Los resultados de esta investigación son concordantes a estudios previos realizados en México, Colombia, Perú y Ecuador, de esta manera tenemos que la dependencia al alcohol es menos incidente en mujeres en comparación con los hombres, esto es apoyado por un estudio sobre las Expectativas, consumo de alcohol y problemas asociados en estudiantes universitarios de la ciudad de México25, donde se halló que las mujeres tienen una tendencia hacia un patrón de consumo de alcohol moderado; de la misma manera en el trabajo: Consumo de alcohol en estudiantes universitarios 26 , mostraron que la más alta prevalencia del consumo de alcohol alguna vez en la vida fue en hombres (92.9\%), mientras que en las mujeres se encontró un $86 \%$. En el artículo: Consumo patológico de alcohol entre los estudiantes de la Universidad de Cartagena27, en cuanto al sexo se encuentra resultados que también apoyan la idea que el consumo de alcohol de alto riesgo es mayor en hombres $(43.8 \%)$ que en mujeres $(27.4 \%)$.

Sobre la adicción a sustancias, el III Estudio epidemiológico andino sobre consumo de drogas en la población universitaria de Ecuador, 201628, coincide con nuestros resultados en el sentido que señala que la tasa de usos de cualquier droga llegó a un $18.3 \%$ entre los estudiantes hombres del Ecuador, mientras que para las mujeres fue de $7.5 \%$.

El artículo sobre El juego de azar y el videojuego en la universidad de San buenaventuraMedellín29, concluye sobre el juego patológico que, el 80\% de los universitarios juegan dinero con mayor o menor frecuencia, existiendo un 4,5\% de probables jugadores patológicos y unos 6,6\% de universitarios considerados jugadores problema. El 90,9\% de los jugadores patológicos 
son varones, mientras que el 74,9\% de los considerados no jugadores son mujeres, estableciéndose una relación inversa muy significativa. De la misma manera, en el trabajo Juego patológico y dependencia del alcohol en una muestra de trabajadores y estudiantes universitarios: prevalencias, interrelaciones y diferencias de género30, expone que en cuanto, al sexo, la proporción de mujeres es menor en los perfiles que presentan alta dependencia del alcohol, y alta adicción al juego.

La adicción a los videojuegos es fuerte entre los universitarios, prevaleciendo en hombres esto lo apoya el estudio Influencia de los videojuegos en los estudiantes de educación superior31, donde se indica que existe una enorme disparidad de género en la cantidad de tiempo dedicado a los videojuegos. Mientras que menos de 1 de cada 50 mujeres encuestadas jugaron más de 10 horas de videojuegos por semana, 1 de cada 10 hombres admitió haber hecho esto. También, son 10 veces más hombres que mujeres los que admiten jugar más de 20 horas por semana.

La adicción al Internet en este estudio no mostro un patrón claro sobre la prevalencia en hombres o mujeres, sin embargo, el trabajo: Adicción a Internet32, señala que las mujeres están más propensas a tener un nivel de adicción mayor al de los hombres, ya que estas acceden al internet con el propósito de ampliar su círculo social, sin embargo, los hombres acceden a páginas con algún contenido de sexo con mayor frecuencia.

En lo referente a gasto compulsivo, fue más prevalente en mujeres en el presente trabajo, esta conducta adictiva es apoyada por el trabajo Impulsividad en la compra en estudiantes universitarios33, en el que, con relación al sexo las medias de la escala impulsividad en las compras presenta diferencia en las medias en función, siendo mayor en las mujeres con un 67,97 en comparación con los hombres con una media de 62,24. Así mismo34, muestra que las mujeres son más propensas al gasto compulsivo.

Finalmente, este estudio no muestra un patrón claro según sexo en el consumo de marihuana, sin embargo, el III Estudio epidemiológico andino sobre consumo de drogas en la población universitaria de Ecuador, 201628, expone que el 39\% de los estudiantes percibe gran riesgo frente al uso experimental (probar marihuana una o dos veces), siendo algo mayor en el caso de las mujeres. Dicha cifra aumenta si los estudiantes son consultados sobre el riesgo percibido cuando una persona fuma frecuentemente marihuana (73\%), en este caso las mujeres aparecen con una percepción de gran riesgo bastante mayor que los hombres, 77,5\% versus $68,2 \%$.

\section{Conclusiones.}

Luego de haber realizado este estudio y mediante el análisis de las variables sexo y adicciones, se llegan a las conclusiones de gran interés siguientes:

- Los estudiantes universitarios de la ESPOCH forman dos perfiles característicos que, en términos generales, el primero se halla representado por el $51.40 \%$ de los estudiantes y está conformado por mujeres $(242,34.6 \%)$ y hombres $(244,99.6 \%)$ casi en la misma cantidad, con alta probabilidad de dependencia al alcohol (78, 61.4\% y 66, 98.5\%), al juego 
patológico $(105,98.1 \%$ y 13, 100\%), adicción a sustancias (39, 100\% y 2, 100\%), videojuegos $(81,96.4 \%, 40,100 \%$ y $19,100 \%)$ y al sexo $(37,100 \%$ y $17,100 \%)$. Por otro lado, también la conforman aquellos estudiantes que tienen segura o nula existencia de dependencia hacia la marihuana $(13,100 \%$ y $253,48.4 \%)$, gasto compulsivo $(54,100 \%$ y $313,62 \%)$ y adicción a internet $(45,95.7 \%$ y $188,100 \%)$. Desde otro punto de vista, el perfil incluye a individuos que no tiene prevalencia $(403,100 \%)$ a sufrir trastornos de alimentación y otros que tienen posible existencia de este trastorno $(58,29.4 \%)$.

- El segundo perfil, fue conformado por 459 (48.6\%) estudiantes de sexo femenino (458), con tendencia alta de sufrir trastornos de alimentación (139, 70.6\%; 304, 93\% y 16, 88.9\%), asimismo está conformada por aquellos que tienen una posible o muy probable predisposición a sufrir un desorden de gasto compulsivo (178, 70.4\% y 89, 66.9\%), adicción a internet $(220,70.7 \%$ y $237,59.4 \%)$, con inexistencia, posible o muy probable dependencia al alcohol $(189,50.1 \% ; 220,58.8 \%$ y $49,38.6 \%)$ y al consumo de marihuana $(270,51.6 \% ; 177,44.9 \%$ y $12,80 \%)$. En contraste, este grupo se ve poco afectado por el juego patológico $(457,55.4 \%$ y $2,1.9 \%)$, adicción a sustancias $(382,47.2 \%$ y $77,81.9 \%)$, videojuego $(456,56.9 \%$ y $3,3.6 \%)$ y al sexo $(459,51.5 \%$ y $0,0 \%)$. Este perfil permite mostrar conclusiones muy interesantes como: que las mujeres son más propensas a sufrir trastornos alimenticios, desorden de gasto compulsivo y adicción al internet, en menor medida son propensas a ser dependientes del alcohol y al consumo de marihuana, y son mesuradamente perjudicadas por el juego patológico, adicción a sustancias, videojuegos y adicción al sexo.

\section{Referencias bibliográficas.}

Dominguez Lara, S. A., Villegas García, G., \& Centeno Leyva, S. B. (2014). Procrastinación académica: validación de una escala en una muestra de estudiantes de una universidad privada. Liberabit, 20(2), 293-304.

Ramos-Galarza, C., Jadán-Guerrero, J., Paredes-Núñez, L., Bolaños-Pasquel, M., \& GómezGarcía, A. (2017). Procrastinación, adicción al internet y rendimiento académico de estudiantes universitarios ecuatorianos. Estudios pedagógicos (Valdivia), 43(3), 275-289.

Salgado, N. L., Aldana, L. A. Q., \& Gaitán, L. C. V. (2018). Consumo de alcohol en estudiantes de la Universidad de la Amazonia. Drugs and Addictive Behavior, 3(2), 219-232.

Palacios Berrospi, G. N. (2018). Uso de las redes sociales y su relación con el desempeño académico. 
Garrote-Rojas, D., Jiménez-Fernández, S., \& Gómez-Barreto, I. M. (2018). Problemas derivados del uso de internet y el teléfono móvil en estudiantes universitarios. Formación universitaria, 11(2), 99-108.

Gómez Cruz, Z., Landeros Ramírez, P., Noa Pérez, M., \& Patricio Martínez, S. (2018). Consumo de alcohol, tabaco y otras drogas en jóvenes universitarios. Revista Salud Pública y Nutrición, 16(4), 1-9.

Osorio-Sánchez, Y. L., Díez-Cardona, P. A., \& Bedoya-Olaya, A. D. (2018). Representaciones sociales sobre consumo de sustancias en contexto universitario: la voz de los estudiantes. Drugs and Addictive Behavior, 3(1), 17-34.

López Machado, H. A. (2019). Construcción de perfiles mediante clúster bietapico sobre el consumo de alcohol y su relación con otras adicciones en la escuela superior politécnica de Chimborazo, octubre 2018-marzo 2019 (Bachelor's thesis, Escuela Superior Politécnica de Chimborazo).

Instituto Nacional de Estadística y Censos, Datos estadísticos sobre el consumo de bebidas alcohólicas en Ecuador, 2012, Disponible en: http://www.ecuadorencifras.gob.ec/documentos/webinec/Revistas/Postdata/postdata01/fil es/assets/downloads/page0006.pdf

Brito Ramos, E. E. (2018). Inteligencia emocional y consumo de alcohol en estudiantes universitarios (Bachelor's thesis, Universidad Técnica de Ambato-Facultad de Ciencias de la Salud-Carrera de Psicología Clínica).

Instituto Nacional de Estadística y Censos SIIH, Formulario de encuesta de condiciones de vida sexta ronda, 2015, Disponible http://www.ecuadorencifras.gob.ec/documentos/webinec/ECV/ECV_2015/documentos/ Metodologia/Formulario\%20ECV\%206R.PDF

Túquerres Chicaiza, J. E., \& Ulcuango Guatemal, L. R. (2019). Estudio del consumo de cigarrillos y de bebidas alcohólicas, en mujeres de edad fértil en el Ecuador, 2014 (Bachelor's thesis, Quito: UCE).

Sierra, V. P., Martínez, J. I. V., González, B. Z., Cárdenas, F. P., Narváez, Y. V., Ramos, L. R., \& Aranda, O. M. (2016). Adicción y factores determinantes en el uso problemático del internet, en una muestra de jóvenes universitarios. Edutec. Revista Electrónica de Tecnología Educativa, (56), a337-a337.

del Castillo, J. A. G., del Carmen Terol, M., Nieto, M., Lledó, A., Sánchez, S., Martín-Aragón, M., \& Sitges, E. (2008). Uso y abuso de Internet en jóvenes universitarios. Adicciones, 20(2), 131-142. 
Muhingi, W. N., Mutavi, T., Kokonya, D., Simiyu, V. N., Musungu, B., Obondo, A., \& Kuria, M. W. (2015). Social Networks and Students' Performance in Secondary Schools: Lessons from an Open Learning Centre, Kenya. Journal of Education and Practice, 6(21), 171-177.

Cazenave, A., Saavedra, W., Huerta, P., Mendoza, C., \& Aguirre, C. (2017). Consumo de marihuana en jóvenes universitarios: percepción de los pares. Ciencia y enfermería, 23(1), $15-24$.

Hernández, R., Fernández, C., \& Baptista, L. (2014). Metodología de la investigación. Sexta edición por McGRAW-HILL.

Patten, M. L., \& Newhart, M. (2017). Understanding research methods: An overview of the essentials. Routledge.

Pérez, E. P., Monje, M. R., Alonso, F. G., Girón, M. F., López, M. P., \& Romero, J. C. (2007). Validación de un instrumento para la detección de trastornos de control de impulsos y adicciones: el MULTICAGE CAD-4. Trastornos Adictivos, 9(4), 269-278.

Marcatoma, J. A. (2016). Estudio del Cannabis y su relación con otras Adicciones en estudiantes de la Universidad de Granada (Tesis de maestría). Universidad de Granada, España.

Van Der Pol, P., Liebregts, N., de Graaf, R., Korf, DJ, van den Brink, W. y van Laar, M. (2013). Predecir la transición del consumo frecuente de cannabis a la dependencia del cannabis: un estudio prospectivo de tres años. Dependencia de drogas y alcohol , 133 (2), 352-359.

Pérez López, C. (2005). Métodos estadísticos avanzados con SPSS. Thompson. Madrid.

Mullo, H., \& Marcatoma, J. (2018). Desempleo juvenil Ecuador. Caribeña de Ciencias Sociales, (marzo).

Míguez, M. C., \& Becoña, E. (2015). Do cigarette smoking and alcohol consumption associate with cannabis use and problem gambling among Spanish adolescents?. adicciones, 27(1).

Mora-Ríos, J., \& Natera, G. (2001). Expectativas, consumo de alcohol y problemas asociados en estudiantes universitarios de la ciudad de México. Salud pública de México, 43, 89-96.

Montaño, M. R., Guarín, A. M., Millán, M. A. G., Maldonado, A. V., \& Díaz, C. A. G. (2011). Consumo de alcohol en estudiantes universitarios. Psicogente, 14(25), 27-35.

Arrieta Vergara, K. M. (2009). Consumo patológico de alcohol entre los estudiantes de la Universidad de Cartagena, 2008. Revista de Salud Pública, 11, 878-886. 
K. Hölge, III Estudio epidemiológico andino sobre consumo de drogas en la población universitaria de Ecuador, 2016. Oficina de las Naciones Unidas Contra la Droga y el Delito (UNODC), 2017.

Pérez, J. A. P., \& Bedoya, V. H. C. (2007). El juego de azar y el videojuego en la universidad de San buenaventura-Medellín. El Ágora USB, 7(2), 273-282.

Pérez, J. I. R. (2014). Juego patológico y dependencia del alcohol en una muestra de trabajadores y estudiantes universitarios: prevalencias, interrelaciones y diferencias de género. Psychologia. Avances de la disciplina, 8(1), 33-42.

Galido, J. B. S., Rodríguez, J. R., \& Flores, J. A. C. (2018). Influencia de los videojuegos en los estudiantes de educación superior. Revista de Investigación en Tecnologías de la Información, 6(11).

Mendoza, Y., \& Méndez, L. (2014). Adicción a Internet. Educación. Handbook T-III, 121-129.

Jurado, D., Sejnaui, P., \& Uribe-Rodríguez, A. F. (2011). Impulsividad en la compra en estudiantes universitarios. Revista de Psicología Universidad de Antioquia, 3(2), 25-38.

Dittmar, H. (2005). Compulsive buying-a growing concern? An examination of gender, age, and endorsement of materialistic values as predictors. British Journal of Psychology, 96(4), 467-491.

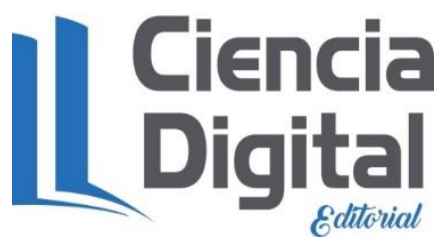


PARA CITAR EL ARTÍCULO INDEXADO.

Marcatoma Tixi, J. A., Mullo Guaminga, H. S., \& Pérez Londo, N. A. (2019). Uso de conglomerados bietapicos para la construcción de perfiles adictivos en estudiantes universitarios. Ciencia Digital, 3(4), 172-188. https://doi.org/10.33262/cienciadigital.v3i4.963

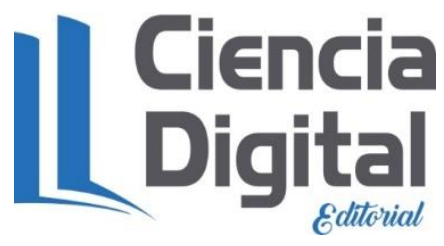

El artículo que se publica es de exclusiva responsabilidad de los autores y no necesariamente reflejan el pensamiento de la Revista Ciencia Digital.

El artículo queda en propiedad de la revista y, por tanto, su publicación parcial y/o total en otro medio tiene que ser autorizado por el director de la Revista Ciencia Digital.
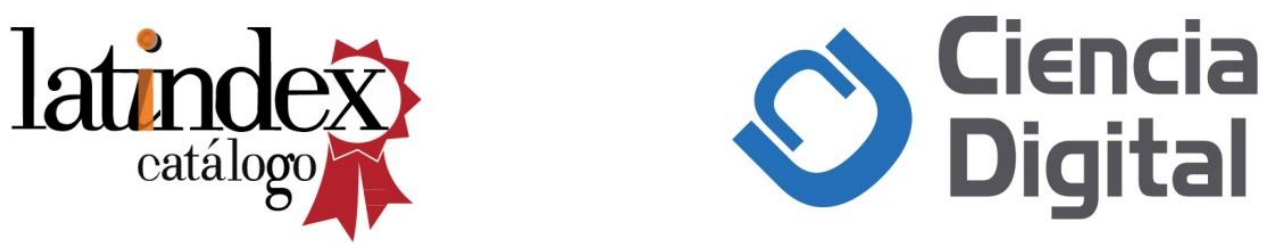